\title{
A influência do capital intelectual na satisfação laboral: um modelo estrutural aplicado a uma cooperativa de crédito
}

The influence of intellectual capital on job satisfaction: a structural model applied to a credit cooperative

\section{Carmem Teresa Leal}

Universidade de Trás-os-Montes e Alto Douro, CETRAD, Quinta de Prados - 5000-801 Vila Real, Portugal, cleal@utad.pt

\section{Carlos Peixeira Marques}

Universidade de Trás-os-Montes e Alto Douro, CETRAD, 5000-801 Vila Real, Portugal, cmarques@utad.pt

\section{Carla Susana Marques}

Universidade de Trás-os-Montes e Alto Douro, CETRAD, 5000-801 Vila Real Portugal, smarques@utad.pt

\section{Elizomar Braga Filho}

UNICRED João Pessoa, UTAD, 58040-140 - João Pessoa, Brasil, elizomarfilho@live.com

\begin{abstract}
Resumo
Dado o atual cenário de instabilidade e incerteza dos mercados, a forma como as empresas conduzem e gerem o seu capital intelectual (CI) é crucial. $\mathrm{O}$ papel do CI tem vindo a ser destacado como o principal motor de desempenho organizacional, devido à sua centralidade no processo de criação de valor, todavia poucos têm sido os trabalhos que analisaram as relações entre CI e a satisfação laboral. Assim, pretendese responder à seguinte questão: como as três dimensões do Capital Intelectual (Capital Humano, estrutural e relacional) são percebidas pelos funcionários da cooperativa de crédito em estudo e quais os respetivos efeitos sobre a satisfação laboral? Nesse sentido, foram traçados como objetivos deste trabalho a determinação do tipo de influência que cada dimensão do CI apresenta sobre a satisfação laboral nesta instituição financeira. É apresentado um modelo de equações estruturais que se debruça sobre as relações entre as dimensões do CI de capital humano, estrutural e relacional - e a satisfação no trabalho. Os dados foram obtidos a partir de um questionário baseado em Bontis (1998) e aplicado a 109 funcionários de uma cooperativa de crédito no Nordeste do Brasil (UNICRED - João Pessoa). Os resultados obtidos sugerem que o capital estrutural tem uma influência sobre a satisfação e desempenhando ainda o papel de mediador entre o capital humano e satisfação laboral. Por outro lado, o capital relacional não exerce nenhuma influência significativa sobre a satisfação.
\end{abstract}

Palavras-chave: Capital Humano, Capital Estrutural, Capital Relacional, cooperativas de crédito, satisfação laboral.

\begin{abstract}
Given the current scenario of marketplace instability and uncertainty the way companies conduct and manage their intellectual capital (IC) is crucial. Numerous studies have highlighted the role of IC as a key driver of organizational performance due to its centrality as an asset in the value creation process, but few have analysed the relationships between IC and the staff satisfaction. Thus, we intend to answer the following question: how the three dimensions of intellectual capital (human capital, structural and relational) are perceived by credit union employees and which is the respective effect on job satisfaction? In this sense, it was pretended to determine the influence of each IC's dimension on job satisfaction in this financial institution. This paper presents a structural model of the relationships linking the dimensions of IC - human, structural, and relational capital - to job satisfaction. In order to evaluate this model, empirical data was obtained from a questionnaire based on Bontis (1998) and applied to 109 employees of a credit cooperative in northeaster Brazil (UNICRED - João Pessoa). Results suggest that structural capital has a close influence over satisfaction and also plays the role of mediator between human capital and satisfaction. On the other hand, relational capital exerts no significant influence over satisfaction.
\end{abstract}

Keywords: Human Capital, Structural Capital, Relational Capital, Credit unions, Job satisfaction.

\section{Introdução}

Devido à sua importância para o sucesso sustentado das empresas, tem emergido uma corrente de literatura que aborda o conteúdo do Capital Intelectual como uma fonte de vantagem competitiva nos dias de hoje. Um novo paradigma surgiu, marcado pela transição de uma sociedade industrial para a sociedade do conhecimento.

Os ativos fixos, facilmente quantificáveis e valorizados pela contabilidade, não são mais a principal fonte de criação de vantagem competitiva (Tejedo-Romero e Tejada-Ponce, 2013). De acordo com Madhani (2009) os ativos intangíveis são os principais vetores de desempenho na transição de um modelo tradicional para uma economia baseada no conhecimento, sendo responsáveis pela criação de valor da empresa.
Ativos, tais como a fidelidade à marca, a cultura organizacional, ou a motivação e compromisso do pessoal são difíceis ou quase impossíveis de replicar e representam uma parte crescente do valor das empresas. Entre esses intangíveis: o conhecimento, em seu sentido mais amplo, aparece como um catalisador na busca para alcançar a sustentabilidade da vantagem competitiva da empresa, claramente relacionada à sua capacidade de inovação (Youndt, Subramaniam e Snell, 2004; Subramaniam e Youndt, 2005).

O conceito de Capital intelectual tem sido utilizado para representar esses ativos intangíveis, que foram classificados em três componentes distintas: humana, estrutural e relacional (Bontis, 1998). Embora Bontis e Serenko (2009) já tenham estudado o impacto do Capital Intelectual em cooperativas de crédito no Canadá, as suas proposições eram diferentes das nossas. Sublinha-se, neste caso, a 
relevância atual do CI nas organizações de serviços financeiros, principalmente no setor bancário, tentando contribuir para a compreensão do seu papel numa cooperativa de crédito brasileira, nomeadamente quanto ao impacto que tem sobre a satisfação laboral dos seus funcionários.

Pretende-se, portanto, responder a esta questão nuclear: como é que as três dimensões do Capital Intelectual (Capital Humano, estrutural e relacional) são percebidas pelos funcionários da instituição e quais os respetivos efeitos sobre a satisfação no trabalho? Assim, na próxima seção alguma literatura sobre o capital intelectual e satisfação no trabalho será brevemente revista; posteriormente, e após a apresentação do modelo de pesquisa, o procedimento metodológico adotado será explicado, e os resultados da sua validação empírica descrita; finalmente, apresentar-se-ão as conclusões, implicações e limitações da pesquisa.

\section{Revisão de Literatura e Modelo de Investigação}

\subsection{Capital Intelectual}

Marr (2008) afirma que o CI, em conjunto com o capital físico e financeiro, é um dos três recursos vitais das organizações. Segundo o autor o CI inclui todos os recursos intangíveis que: (a) pertencem a uma organização, e (b) contribuem para a criação de valor.

Martins, Morais e Isidro (2012) argumentam que o CI pode ser explicado pelo valor que os investidores atribuem aos ativos intangíveis que não podem ser avaliados de forma precisa, mas têm potencial para criar valor para os investidores no futuro.

Desde o final do século passado até os dias atuais, muito se tem escrito sobre os ativos incorpóreos das empresas e sua respetiva importância (Guthrie; Ricceri e Dumay 2012; Cañibano; García-Ayuso e Sanchez 2000; Dumay, 2009; Skinner, 2008; Wyatt, 2008), no entanto, e de acordo com Martins et al. (2012) e Tejedo-Romero e Tejada-Ponce (2013), mesmo ao nível internacional, não existem condições consistentes na divulgação do CI pelas empresas, sendo muito poucas aquelas que praticam uma divulgação voluntária de investimentos nesse ativo intangível.

Apesar disso, tanto ao nível académico como ao nível profissional a importância do CI tem sido reconhecida, como uma fonte de geração de vantagem competitiva (Martins et al. 2012). Longo e Mura (2011) afirmam, ainda, que a última década tem sido vista como um momento de desmaterialização dos recursos estratégicos das empresas. Neste sentido, a produtividade e o desempenho organizacional têm vindo a depender em grande parte da gestão eficiente desses recursos (Chen, Shih e Yang, 2009; Kang e Snell, 2009; Campisini e Costa, 2008; Reed, Lubatkin e Srinivasan, 2006; Subramaniam e Youndt, 2005; Sveiby, 1997).

Falar de conhecimento nas organizações é falar de CI, apesar de não serem sinónimos, uma vez que o conceito de CI é muito mais amplo do que o conhecimento por si só. Kaplan e Norton (2004) definem Capital Intelectual como um conjunto de técnicas, talentos e áreas específicas de conhecimento detido pelos recursos humanos das organizações. A Organização para a Cooperação e
Desenvolvimento Económico (OCDE, 2008) define Capital Intelectual como um recurso intangível utilizado na criação de valor futuro, e que inclui a propriedade intelectual, o capital relacional, o capital humano e o capital organizacional. Neste sentido, parece existir na literatura um consenso quanto à estrutura tridimensional do CI: capital humano, estrutural e relacional.

Capital humano: Com base em Lee (2010) e Youndt et al. (2004) considera-se que o capital humano inclui os recursos organizacionais chave, tais como habilidades, atitudes, experiência e motivação, características de liderança e habilidades intelectuais, como o conhecimento, a inovação ou adaptação. Para Kraft e Lang (2013) e Edvinsson e Malone (1997) o capital humano emerge como o acúmulo de investimento em formação. Deve notar-se que este tipo de capital é perdido quando os funcionários saem das empresas (Boohene e Asuinura, 2011). 0 conhecimento é um ativo que não pode ser expropriado (Granstrand e Holgersson, 2013) e saber como controlá-lo é contribuir para a criação e sustentabilidade do valor criado pelas organizações. Smith (2001) afirma que o capital humano inclui o conhecimento coletivo, a criatividade e a inovação em instituições. Habilidades de liderança, espírito de equipa, competências, nível de investimento na formação de recursos humanos, baixa taxa de rotatividade dos funcionários, número de funcionários em dedicação exclusiva, entre outras, podem ser exemplos disso mesmo (Wright et al. 2014).

Capital relacional: Ross, Ross, Edvinsson, e Dragonneti (1997) argumentam que o conhecimento também está incluído nas relações com os stakeholders externos, como clientes, fornecedores e parceiros. De acordo com Capello e Faggian (2005) o capital relacional consiste em todas as relações - de troca, de poder e de cooperação - que se estabelecem entre as empresas, as instituições e as pessoas, resultante de um forte sentimento de pertença e uma capacidade altamente desenvolvida para colaboração.

Capital Estrutural: engloba todos os processos, sistemas, estruturas, marcas, propriedade intelectual e outros bens incorpóreos da empresa não refletidos em suas demonstrações contabilísticas (Pandey e Dutta 2013). De acordo com Ross et al. (1997) alguns elementos da Capital estrutural podem ser legalmente protegidos através de patentes que preservam os resultados da investigação e desenvolvimento das empresas. Edvinsson e Sullivan (1996) afirmam que, diferentemente do Capital Humano, o Capital estrutural é um bem intangível que pode ser trocado, compartilhado ou reproduzido. Assim, em empresas de conhecimento, o capital estrutural devem ser projetado para maximizar a produção intelectual.

OCDE (2008), Lee et al. (2005), Youndt et al. (2004), Bontis (1998), Lynn (1998), Dzinkowski (2000); Wall (2005) e Kristandl e Bontis (2007) destacam a interação desses três dimensões no processo de criação de valor dentro das organizações. Além disso, alguns autores argumentam sobre as relações causais entre as três dimensões. Por exemplo, Bontis e Fitz-enz (2002) apresentaram um modelo onde o capital humano era influenciado pelo capital estrutural e pelo capital relacional. Da mesma forma, Moon 
e Kym (2006) e Ordóñez de Pablos (2004) apresentaram uma ligação entre o capital humano e as outras duas dimensões. Finalmente, Longo e Mura (2011) consideraram o capital humano como elemento principal do capital intelectual, contribuindo para as outras duas dimensões sublinhando ainda que o capital estrutural é afetado tanto pelo capital humano e como pelo capital relacional. Desta forma, propomos:

H1: O Capital Humano influencia positivamente o Capital Estrutural.

$\mathbf{H}_{2}$ : O Capital Humano influencia positivamente o Capital Relacional.

$\mathbf{H}_{3}$ : O Capital Estrutural influencia positivamente o Capital Relacional

\subsection{Capital Intelectual e a Satisfação no Trabalho}

Jorfi e Jorfi (2011) definem a satisfação no trabalho como uma atitude que muda ao longo do tempo. Para esses autores os fatores de satisfação e insatisfação laboral não são constantes, porque reações afetivas do funcionário. Fatores como a formação, promoções, avaliação de desempenho ou salários (aumento) são apontados como causadores de satisfação laboral (Ealias e George, 2012).

As organizações que primeiro compreendam que, mantendo um contingente de trabalhadores satisfeitos, estáveis, produtivos e diligentes, obterão uma redução nos seus custos e aumento dos lucros. Assim, e assumindo que funcionários satisfeitos tornam-se comprometidos com os interesses da organização, provavelmente atingirão níveis de produtividade, eficácia e eficiência muito maiores. Além disso, sabendo que os recursos humanos são o único ativo que não pertence à empresa, e que uma alta taxa de rotatividade do pessoal representa horas gastas em formação e preparação completamente perdidas, tudo isso em conjunto, revela a importância que a questão da satisfação dos recursos humanos qualificados e preparados representa para as empresas no século XXI.

Inúmeros estudos têm destacado a capacidade do CI como um promotor do desempenho organizacional (Chen et al, 2009; Kang e Snell, 2009; Campisini e Costa, 2008; Reed et al., 2006; Subramaniam e Youndt 2005; Sveiby, 1997). No entanto, existem poucos estudos que demonstrem a relação que pode eventualmente existir entre as dimensões do Capital Intelectual e a satisfação no trabalho (Longo e Mura, 2011).

Por exemplo, Longo e Mura (2011) demonstraram, num estudo aplicado à indústria alimentar italiana, um efeito positivo direto sobre a satisfação com o trabalho causado pelo Capital Intelectual. Por sua vez, Campos, Mendes, Silva e Valle (2014) encontraram uma influência indireta do capital humano na satisfação laboral de 128 unidades no sector do Turismo no sul de Portugal. Assim, propõe-se:

H4: O Capital Humano influencia positivamente a satisfação laboral na UNICRED-JP

H5: O Capital estrutural influencia positivamente a satisfação laboral na UNICRED-JP

H6: O Capital Relacional influencia positivamente a satisfação laboral na UNICRED-JP

Com base na revisão de literatura apresenta-se o modelo conceptual (Figura 1) que reflete a premissa base deste trabalho em conjunto com as hipóteses de trabalho já definidas:

Figura 1 - Modelo Concetual de Investigação

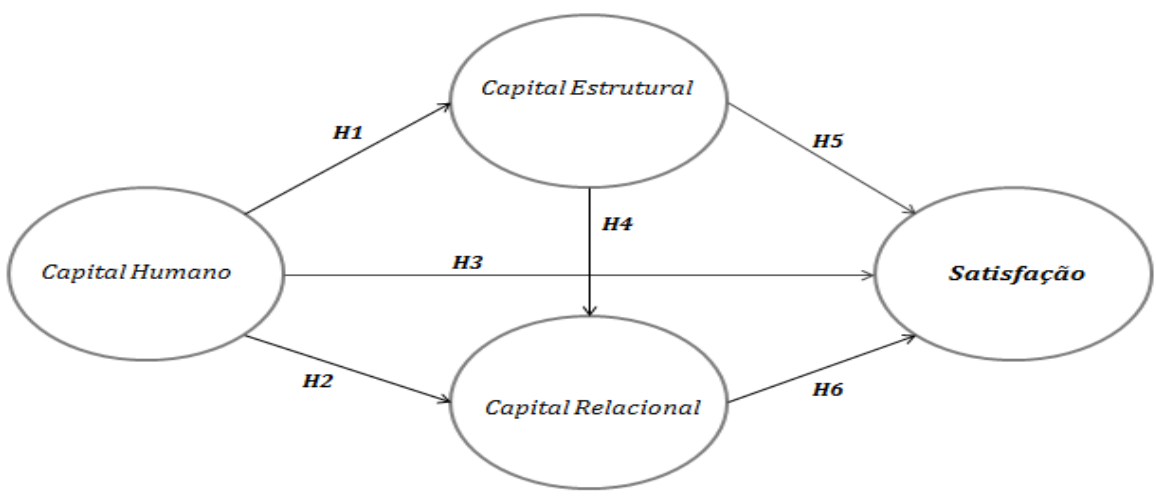

Fonte: Elaboração Própria.

\section{Método - População, amostra e procedimentos}

0 trabalho que aqui se apresenta foi realizado sob um prisma de um estudo de caso, na medida em que se debruçou sobre uma cooperativa de crédito, dando origem a um estudo exploratório com uma vertente quantitativa, baseado num inquérito por questionário à totalidade (109) dos funcionários da UNICRED - JP. Para atingir os objetivos propostos, o questionário foi adaptado ao contexto particular desta CC, a partir do trabalho de Bontis (1998), com o propósito de aferir, junto dos recursos humanos da UNICRED de João Pessoa-PB, o modo como os três factores de CI (humano, estrutural e relacional) poderão influenciar a respetiva satisfação laboral. Esta, por sua vez, foi operacionalizada por um item de satisfação global e quatro de satisfação com aspetos relacionados com o fator de motivação, segundo a teoria dualista de Herzberg (1966). Todos os indicadores foram medidos numa escala rotulada de cinco pontos: os itens de CI numa escala de concordância em que 1 corresponde a "discordo totalmente" e 5 a "concordo totalmente"; os itens de satisfação numa escala bipolar em que 1 corresponde a "muito insatisfeito" e 5 a "muito satisfeito".

Utilizou-se o método da máxima verosimilhança a partir da matriz de variância-covariância para avaliar, em primeiro 
lugar, o modelo de medida dos 17 indicadores (quatro de cada uma das dimensões de CI e cinco de satisfação) e, em segundo lugar, o modelo de relações estruturais entre as quatro variáveis latentes. 0 tamanho da amostra, apesar de ser inferior ao habitualmente usado em modelos de equações estruturais, pode ser considerado adequado para um modelo com as características do que aqui é avaliado, com apenas quatro variáveis latentes, todas elas medidas pelo menos por três indicadores (Hair, Black, Babin \& Anderson, 2010). Considerando a proposta de Kim (2005), esta amostra fica aquém do mínimo aconselhado para garantir RMSEA=0,05 em condições de bom ajustamento, sendo adequada para o valor de RMSEA=0,064

\subsection{UNICRED de João Pessoa - Breve Caraterização}

Constituída em Assembleia Geral de 19 de dezembro de 1990 e homologada pelo Banco Central do Brasil em 26 de abril de 1993, a UNICRED João Pessoa é uma instituição financeira formada com uma história de sucesso. Neste momento possui seis agências estrategicamente localizadas para atender os seus cooperados que hoje se cifram em mais de 9.300 membros. Esse crescimento bastante expressivo do número de cooperados comprova a credibilidade conquistada pela UNICRED JP e que pode ser referendada por outros números representativos: ativos totais na ordem dos $\mathrm{R} \$ 550$ milhões; mais de $\mathrm{R} \$ 400$ milhões na carteira de crédito; Património Líquido de mais de $\mathrm{R} \$ 190$ milhões; mais de $\mathrm{R} \$ 260$ milhões nos depósitos a prazo; e mais de $\mathrm{R} \$ 60$ milhões nos depósitos à ordem.

\section{Resultados}

As medidas de CI e de satisfação laboral são apresentadas na Tabela 1. As médias de todos os indicadores são bastante elevadas, situando-se no intervalo de 3,6 (autonomia) a 4,8 (relação com os cooperados). Analisando as médias das variáveis latentes, é de realçar o valor extremamente elevado da perceção de CR, considerando os colaboradores que a relação com os cooperados é muito boa. A média mais baixa é obtida pela medida de satisfação, correspondendo ao ponto 4 da escala, que está rotulado "satisfeito". Avaliando o modelo de medida através de análise fatorial confirmatória, o ajustamento do modelo pode ser considerado, de acordo com Marôco (2010), bom para alguns índices de qualidade $\left(\chi^{2} /\right.$ g.l. $=1,786$; RMSEA=0,085), mas sofrível para outros (GFI=0,828; $\mathrm{CFI}=0,896$ ). Para aumentar a qualidade de ajustamento, foram retirados do modelo um item de cada fator de $\mathrm{CI}$, com base não só nos respetivos pesos fatoriais, mas também nos índices de modificação, os quais indicavam que os itens "confiança entre UNICRED e colaboradores" e "cooperados informados" eram afetados por outras variáveis.

Tabela 1 - Modelos de medida

\begin{tabular}{|c|c|c|c|c|c|c|}
\hline \multirow[b]{2}{*}{ Item (dimensão) } & \multirow[b]{2}{*}{ Média } & \multirow[b]{2}{*}{ DP } & \multicolumn{2}{|c|}{ Modelo 17 itens } & \multicolumn{2}{|c|}{ Modelo 14 itens } \\
\hline & & & peso fatorial & VE & VEM & FC \\
\hline Humano & 4,200 & & 0,836 & 0,565 & 0,830 & 0,626 \\
\hline Sugerir ideias & 4,202 & ,847 & 0,893 & 0,797 & 0,902 & 0,814 \\
\hline Dar opinião & 4,156 & ,964 & 0,793 & 0,629 & 0,839 & 0,704 \\
\hline Confiança colaboradores & 4,431 & 685 & 0,661 & 0,437 & & \\
\hline Iniciativa colaboradores & 4,009 & ,877 & 0,630 & 0,397 & 0,601 & 0,361 \\
\hline Estrutural & 4,333 & & 0,803 & 0,509 & 0,785 & 0,554 \\
\hline Oportunidade de aprendizagem & 4,358 & ,764 & 0,808 & 0,653 & 0,865 & 0,748 \\
\hline Formação de acordo com objetivos & 4,394 & ,828 & 0,786 & 0,618 & 0,748 & 0,560 \\
\hline Troca de conhecimentos & 4,394 & ,707 & 0,631 & 0,398 & 0,596 & 0,355 \\
\hline Colaboradores excelentes & 4,183 & ,807 & 0,605 & 0,366 & & \\
\hline Relacional & 4,617 & & 0,749 & 0,428 & 0,708 & 0,451 \\
\hline Relação com cooperados & 4,798 & ,426 & 0,713 & 0,508 & 0,78 & 0,608 \\
\hline Necessidades dos cooperados & 4,752 & ,434 & 0,676 & 0,457 & 0,616 & 0,379 \\
\hline Cooperados informados & 4,642 & 601 & 0,623 & 0,388 & & \\
\hline Produtos diferenciados & 4,275 & ,837 & 0,600 & 0,360 & 0,604 & 0,365 \\
\hline Satisfação & 4,013 & & 0,869 & 0,571 & 0,868 & 0,569 \\
\hline Satisfação global & 4,156 & 611 & 0,802 & 0,643 & 0,801 & 0,642 \\
\hline Desafio intelectual & 4,055 & ,718 & 0,800 & 0,640 & 0,792 & 0,627 \\
\hline Responsabilidade & 4,174 & 678 & 0,778 & 0,605 & 0,784 & 0,615 \\
\hline Contributo para a comunidade & 4,046 & ,821 & 0,699 & 0,489 & 0,698 & 0,487 \\
\hline Autonomia & 3,633 & ,889 & 0,690 & 0,476 & 0,689 & 0,475 \\
\hline
\end{tabular}

Após a retirada destes três indicadores, os índices de qualidade apontam para um ajustamento bom: $\chi^{2} /$ g.l. $=1,43$; GFI=0,895; $\quad \mathrm{CFI}=0,955$; $\quad$ RMSEA=0,063; $p($ RMSEA $\leq 0,05)=0,217$. A fiabilidade compósita das medidas diminuiu ligeiramente, enquanto a variância extraída média aumentou, como seria de esperar. No modelo de medida final, todas as variáveis latentes são fiáveis ( $\mathrm{FC}>0,7)$, mas a variável CR sofre de falta de validez convergente $(\mathrm{VEM}<0,5)$, ou seja, os itens desta escala têm menor comunalidade, sendo demasiado afetados por erro de medida. A utilização de CR no modelo da Figura 1 deve ter em conta esta limitação, ou seja, deve ser considerada a 
possibilidade destes itens não medirem corretamente a perceção de CR nos colaboradores da UNICRED, pelo que a estimação das relações com as restantes variáveis do modelo pode ser enviesada.

Na Tabela 2 pode verificar-se que as médias dos pesos fatoriais são superiores a todas as correlações entre as variáveis latentes, condição necessária para se verificar a validez discriminante, podendo assim concluir-se que as três facetas de CI não só são distintas da medida de satisfação, como são também distintas entre si, permitindo conceptualizar o $\mathrm{CI}$ como constructo tridimensional.

Tabela 2 - Correlações entre as variáveis latentes

\begin{tabular}{|l|c|c|c|c|}
\hline & Humano & Estrutural & Relacional & Satisfação \\
\hline Humano & $\mathbf{0 , 7 9 1}$ & & & \\
\hline Estrutural & 0,604 & $\mathbf{0 , 7 4 5}$ & & \\
\hline Relacional & 0,431 & 0,286 & $\mathbf{0 , 6 7 1}$ & \\
\hline Satisfação & 0,518 & 0,721 & 0,315 & $\mathbf{0 , 7 5 4}$ \\
\hline Os valores na diagonal são as médias dos pesos fatoriais.
\end{tabular}

Fonte: Elaboração Própria.

No que respeita à estrutura de relações entre as dimensões de CI e entre estas e a satisfação laboral, seguindo as

Tabela 3 - Coeficientes padronizados obtidos por estimativa bootstrap

\begin{tabular}{|c|c|c|c|c|c|}
\hline Caminho & Efeito & 1 & p & I & p \\
\hline$H$-.> E & Direto & 0,604 & 0,001 & 0,628 & 0,001 \\
\hline \multirow[t]{2}{*}{$H-->R$} & Direto & 0,406 & 0,019 & & \\
\hline & Indireto & 0,011 & 0,875 & & \\
\hline \multirow{2}{*}{ H --> Sat } & Direto & 0,093 & 0,567 & & \\
\hline & Indireto & 0,272 & 0,000 & 0,462 & 0,000 \\
\hline$E->R$ & Direto & 0,041 & 0,899 & & \\
\hline \multirow[t]{2}{*}{ E --> Sat } & Direto & 0,638 & 0,001 & 0,736 & 0,001 \\
\hline & Indireto & 0,003 & 0,450 & & \\
\hline R --> Sat & Direto & 0,093 & 0,470 & & \\
\hline
\end{tabular}

Fonte: Elaboração Própria.

Com base nos resultados apresentados na Tabela 3, facilmente se conclui que existe um efeito muito forte, nesta amostra, da dimensão Capital Estrutural, ou seja, tudo que tenha a ver com as rotinas organizacionais, a eficiência das estruturas, a cultura institucional (Bontis, 1998), sobre a satisfação laboral. 0 Capital Humano, ou seja o conhecimento coletivo, a criatividade e inovação das pessoas nas instituições (Sundac e Krmpotic, 2009), tem um orientações de Shrout e Bolger (2002) para modelos de mediação, os efeitos diretos e indiretos correspondentes ao modelo concetual foram avaliados com base nas estimativas dos respetivos coeficientes obtidas em 2000 amostras bootstrap (Tabela 3). 0 modelo de partida, correspondendo a todas as trajetórias diretas desenhadas na Figura 1, tem, obviamente, os mesmos índices de ajustamento do modelo de medida, já que estabelece relações entre todos os pares de variáveis latentes. No entanto, como é visível na Tabela 3 , apenas o CE tem efeito direto significativo sobre a Satisfação Laboral. Por outro lado, o CR depende apenas do $\mathrm{CH}$, não se verificando a esperada dependência do CE.

Atendendo ao mencionado problema de validez convergente do $\mathrm{CR}$, é possível que as trajetórias não significativas tenham sido subestimadas. De qualquer modo, esta dimensão de CI torna-se inútil para explicar a satisfação laboral, reduzindo-se o modelo à estrutura sequencial $\mathrm{CH} \rightarrow \mathrm{CE} \rightarrow$ Satisfação. Este modelo simplificado (modelo final na Tabela 3), apesar de não estabelecer uma trajetória direta entre $\mathrm{CH}$ e Satisfação, tem um ajustamento melhor do que o modelo inicial: $\chi^{2} /$ g.l. $=1,383$; GFI=0,922; $\mathrm{CFI}=0,972 ;$ RMSEA $=0,06 ; \mathrm{p}(\mathrm{RMSEA} \leq 0,05)=0,319$.

\section{Tabela 4 - Hipóteses Testadas - Resultados}

\begin{tabular}{|l|l|}
\multicolumn{1}{|c|}{ Hipóteses } & Validação \\
\hline \begin{tabular}{l|l|}
\hline H1: O Capital Humano influencia positivamente o Capital \\
Estrutural.
\end{tabular} & Validada \\
\hline $\begin{array}{l}\text { H: O Capital Humano influencia positivamente o Capital } \\
\text { Relacional. }\end{array}$ & Validada \\
\hline $\begin{array}{l}\text { H3: O Capital Humano influencia positivamente a } \\
\text { satisfação dos RH da UNICRED - JP. }\end{array}$ & Efeito Indireto \\
\hline $\begin{array}{l}\text { H4: O Capital Estrutural influencia positivamente o Capital } \\
\text { Relacional. }\end{array}$ & Não Validada \\
\hline $\begin{array}{l}\text { H5: O Capital Estrutural influencia positivamente a } \\
\text { satisfação dos RH da UNICRED - JP. }\end{array}$ & Validada \\
\hline $\begin{array}{l}\text { H6: O Capital Relacional influencia positivamente a } \\
\text { satisfação dos RH da UNICRED - JP. }\end{array}$ & Não Validada \\
\hline
\end{tabular}

Fonte: Elaboração própria.

Estes resultados confirmam, de certa forma, as conclusões sugeridas por Ichniowski, Kochan, Levine, Olson e Strauss efeito indireto, i.e. favorece a satisfação pelo seu papel bastante significativo no desenvolvimento do Capital Estrutural. Por sua vez, o Capital Relacional, tal como foi medido neste estudo, não tem qualquer efeito sobre a satisfação.

Com base nestes resultados, resume-se na Tabela 4 o teste das hipóteses do modelo:

(1996) acerca do efeito direto do investimento em capital estrutural sobre a satisfação laboral. Segundo os autores, 
empresas com uma forte dimensão de Capital Estrutural incluem nos seus projetos estratégicos investimento em trabalho colaborativo e sistemas de aprendizagem e partilha de conhecimentos, sendo, geralmente, fatores relevantes na perceção dos funcionários de qualquer organização. Podemos ainda referir resultados mais atuais obtidos por Mura e Longo (2011) que verificaram uma relação positiva, forte, mas indireta entre o CE e a melhoria da performance dos indivíduos, salientando que estes autores identificaram a melhoria da performance como uma consequência direta do aumento da satisfação laboral.

\section{Conclusões}

Através de um inquérito aos RH de uma cooperativa de crédito brasileira, uma organização que usufrui de características muito particulares e diferenciadoras, nomeadamente ao nível da sua gestão, conseguiu-se neste trabalho avaliar o papel que a gestão/investimento em Capital Intelectual, nas suas três dimensões, Humano, Estrutural e Relacional, desempenha no grau de satisfação laboral.

Esta pesquisa permitiu aferir que o staff desta instituição de crédito perceciona muito favoravelmente a relação com os cooperados (seus clientes). Todavia, esta faceta de capital intelectual não influencia a sua satisfação laboral. Tratandose de capital intelectual desenvolvido nas relações de cooperação com stakeholders externos, os funcionários poderão ter mais dificuldade em perceber a sua relação directa com a satisfação laboral, principalmente se houver aspetos relacionados com o capital interno (humano e relacional) que sejam julgados menos satisfatórios. Tal parece ser o caso, já que a média de satisfação é a mais baixa das quatro variáveis latentes.

Constatamos que, também nesta instituição de crédito, e à semelhança de outros estudos (já aqui citados) realizados em outros sectores de atividade, o principal antecedente da satisfação laboral foi o CE. Percebe-se que os RH valorizam o trabalho colaborativo, os sistemas de partilha de conhecimento e as oportunidades de formação e de aprendizagem. $\mathrm{O} \mathrm{CH}$, aqui operacionalizado pelo incentivo ao envolvimento e iniciativa dos colaboradores, contribui fortemente para o CE e, por mediação deste, para a satisfação.

É necessário ter em consideração algumas limitações deste trabalho, desde logo, a dimensão da amostra e próprio fato de se tratar de um estudo de caso impõe cautelas relativamente à extrapolação dos resultados aqui obtidos. Uma segunda limitação tem que ver com a medida de CR, já que os itens aqui utilizados revelaram-se problemáticos ao nível da validez convergente.

A partir destas limitações, podemos indicar vias para futuras investigações. Começamos por sugerir que se replique este questionário na totalidade dos $\mathrm{RH}$ das UNICRED da região Norte-Nordeste do Brasil de forma a conseguir resultados mais robustos e fundamentados. Este estudo poderia também ser efetuado longitudinalmente, de forma a determinar se os fatores que influenciam a satisfação laboral dos RH serão (ou não) estáveis ao longo do tempo e porquê. Relativamente à medida do CR, sugerimos que seja desenvolvida uma nova escala válida para verificar se a ausência de relação entre esta dimensão e a satisfação laboral se pode dever à fragilidade da medida.

Em jeito de conclusão queremos dizer que o principal propósito deste trabalho é, para além de comprovar os desafios que apresentamos ao início, sobretudo, sensibilizar, influenciar e motivar gestores, administradores e decisores em geral para a importância do investimento na gestão destes ativos que na atualidade constituem o segredo do sucesso das organizações. Porém, para que seja um sucesso sustentado é preciso, em primeiro lugar, tomar consciência da necessidade imperiosa de políticas de investimento em formação, preparação e motivação de todos os envolvidos para gerar o comprometimento de todas as partes na busca do que é melhor para a organização. Só depois desta missão concluída poderemos passar à fase da mensuração e valorização correta, válida e assertiva destes investimentos e dos seus retornos efetivos. Sabendo que, no fundo, se trata de uma questão de custo-benefício, não poderemos, contudo, deixar de considerar que, para que essa relação seja positiva, serão necessárias politicas estratégicas de definição de objetivos e de metas a atingir. Só então com políticas bem delineadas e sustentadas em plano estratégicos corretamente definidos e divulgados entre os interessados, será possível trilhar o caminho do sucesso.

\section{Agradecimentos:}

Os autores deste artigo querem agradecer os contributos recebidos durante a TMS Algarve 2014 - Management Studies International Conference.

Este trabalho é financiado por Fundos Nacionais através da FCT Fundação para a Ciência e a Tecnologia no âmbito do projeto PEstOE/SADG/UI4011/2014.

\section{Referências}

Araújo, E. ; Silva, W. (2011). Cooperativas de crédito: A evolução dos principais sistemas brasileiros com um enfoque em indicadores econômico-financeiros. Revista Contemporânea de Economia e Gestão 9 (1), 117-126.

Boohene, K., Asuinura, E.L. (2011). The effect of human resources management practices on corporate performance: a study of graphic communication group limited. International Business Research, 4(1), 266-272

Bontis, N.; Serenko, A. (2009). A causal model of human capital antecedents and consequents in the financial services industry. Journal of Intellectual Capital, 10(1), 53-69.

Bontis, N. (1998). Intellectual capital: an exploratory study that develops measures and models. Management Decision, 36(2), 63 - 76.

Bontis, N. and Fit-enz, J. (2002). Intellectual capital ROI: a causal map of human capital antecedents and consequents, Journal of Intellectual Capital, 3(3), .223 - 247

Campisini, D.; Costa, R. (2008). A DEA-Based Method to Enhance Intellectual Capital Management. Knowledge and Process Management, 15(3), 170-183.

Campos, A., Mendes, J., Silva, J.; Valle, P. (2014). Critical success factors for a total quality culture: A structural model. Tourism \& Management Studies, 10(1), 7-15.

Cañibano, L., García-Ayuso, C.M., Sánchez, P. (2000), Accounting for intangibles: a literature review. Journal of Accounting Literature, 19, (3)102-130.

Capello R., Faggian A. (2005). Collective learning and relational capital in local innovation processes, Regional Studies 39 (1), 75-87.

Chen, S., Yang Y. (2009). The role of intellectual capital in knowledge transfer, IEEE Transactions on Engineering Management 56 (3), 402-411.

Choi B., Lee H. (2003). An empirical investigation of KM styles and their effect on corporate performance, Information and Management 40(5), 403-417. 
DiPiazza, S., Eccles, R. (2002). Recuperar la confianza: el futuro de la información corporativa. Madrid: FT Prentice-Hall.

Dumay, J. C. (2009). Intellectual capital measurement: a critical approach. Journal of Intellectual Capital, 10(2), 190-210.

Dzinkowski, R. (2000). The measurement and management of intellectual capital: an introduction. Management Accounting, 78(2), 32-36.

Edvinsson, L., Sullivan, P. (1996). Developing a model for managing intellectual capital. European Management Journal, 14(4), 356-364.

Edvinsson, L., Malone, M. (1997). Intellectual Capital: Realizing Your Company's True Value by Finding Its Brainpower Hidden. New York: Harper Business.

Ealias, A., George, J. (2012). Emotional intelligence and job satisfaction: a correlational study. Research Journal of Commerce and Behavioral Science, 1(4).

Ferreira, A., Martinez, L. (2011). Intellectual Capital: Productivity and Investment Perceptions, RAC, 15(2), 249-260.

Fontes-Filho, J., Marucci, J., Oliveira, M. (2008). Governança cooperativa: participação e representatividade em cooperativas de crédito no Brasil. Revista de Contabilidade e Organizações, 2(4), 107 - 125

Granstrand, 0.; Holgersson, M. (2013). Managing the intellectual property disassembly problem. California Management Review, 55(4), 184-210.

Greco, M., Cricelli, L., Grimaldi, M. (2013). A strategic management framework of tangible and intangible assets. European Management Journal, 31(1), 55-66.

Guthrie, J., Ricceri, F., Dumay, J. (2012). Reflections and projections: a decade of intellectual capital accounting research. The British Accounting Review, 44(2), 68-82.

Hair, J. F., Black, W. C., Babin, B. J., Anderson, R. E. (2010). Multivariate data analysis (7th ed.). Upper Saddle River, N.J.; Harlow: Pearson Education.

Herzberg, F. I. (1966). Work and the nature of man. Oxford: World.

Ichniowski, C., Kochan, T., Levine, D., Olson C., Strauss, G. (1996). What works at work: overview and assessment, Industrial Relations, 35, (3) 299-333.

Kang, S., Snell A. (2009). Intellectual capital architectures and ambidextrous learning: a framework for human resource management, Journal of Management Studies 46 (1), 65-92.

Kaplan, R. S., Norton, D. P. (2004). Strategy maps: converting assets into tangible outcomes. Boston: Harvard Business School Press.

Kim, K. H. (2005). The relation among fit indexes, power, and sample size in structural equation modeling. Structural Equation Modeling, 12(3), 368-390.

Kraft, K.; Lang, J. (2013). Profit Sharing and Training. Oxford Bulletin Of Economics And Statistics, 75(6), 940-961, John Wiley and Sons.

Kristandl, G., Bontis, N. (2007). The impact of voluntary disclosure on cost of equity capital estimates in a temporal setting. Journal of Intellectual Capital, 8(4), 577-594.

Jorfi H., Jorfi S. (2011). Strategic Operations Management: Investigating the Factors Impacting Communication Effectiveness and Job Satisfaction, in Procedia - Social and Behavioral Sciences, Kulalampur, 1596-1605.

Lee, S. (2010). Using fuzzy AHP intellectual capital evaluation model for assessing their performance contribution in a university. Expert Systems with Application Journal 37, (7), 4941-4947.

Lee, K. Lee, S., Kang, I. (2005). KPMI: measuring knowledge management performance. Information and Management 42, (1) 469-482.

Longo, M., Mura, M. (2011). The effect of intellectual capital on employees' satisfaction and retention. Information and Management 48, (7), 278-287.

Lynn, B. (1998). Intellectual capital. CMA Management, 72(1), 10-15.

Madhani, P. M. (2009). Management of Intangible Assets: A Value Enhancing Strategy" in Knowledge Economy. Intangible Assets: Measurement and Accounting Practices, Pankaj M Madhani, ed., pp. 20-36, ICFAI University Press, Hyderabad.

Marr, B. (2008). Impacting future value: how to manage your intellectual capital. Management Accounting Guideline (MAG), jointly published by CMA, AICPA and CIMA.
Martinez-Torres, M. (2006). A procedure to design a structural and measurement model of intellectual capital: an exploratory study, Information and Management 43 (5), 617-626.

Martins, M., Morais, A., Isidro, H. (2013). O valor do Capital Intelectual das empresas portuguesas. Proceedings of the International International Conference 2012: Financial Management, Accounting and Taxation. Faro: Universidade do Algarve, 848 - 862.

Moon, Y. J., and Kym, H. G. (2006). A Model for the Value of Intellectual Capital. Canadian Journal of Administrative Sciences, 23(3), 253-269.

Nonaka I. and Takeuchi, H. (1996). The knowledge-creating company: How Japanese companies create the dynamics of innovation, New York: Oxford University Press.

OECD. (2008). Measurement, reporting and valuation of intellectual assets - the investor view, Washington DC: OECD Experts Workshop.

Ordóñez de Pablos, P. (2004). Human resource management systems and their role in the development of strategic resources: empirical evidence. Journal of European Industrial Training. 28(6), 474-489.

Pandey, C. Dutta, A., (2013). Role of knowledge infrastructure capabilities in knowledge management. Journal of Knowledge Management 17(3), 435-453

Pinheiro, M. (2005). Cooperativas de Crédito: história da evolução normativa no Brasil. Brasília: Banco Central do Brasil.

Reed, K. Lubatkin, M., and Srinivasan, N. (2006). Proposing and testing an intellectual capital-based view of the firm, Journal of Management Studies 43(4), 867-893.

Roslender, R., Stevenson, J. and Kahn, H. (2006). Employee wellness as intellectual capital: an accounting perspective, Journal of Human Resource, Costing and Accounting 10 (1), 48-64.

Ross, J., Ross, G., Edvinsson, L. and Dragonneti, N. (1997). Intellectual Capital: Navigating in the New Business Landscape. London: Macmillan Business.

Skinner, D. J. (2008). Accounting for intangibles - A critical review of policy recommendations. Accounting and Business Research, 38(3), 191-204

Shrout, P., and Bolger, N. (2002). Mediation in experimental and nonexperimental studies: New procedures and recommendations. Psychological Methods, 7(4), 422-445.

Smith (2001). The role of tacit and explicit knowledge in the workplace. Journal of Knowledge Management 5(4), 311-321.

Subramaniam, M. and Youndt, M. (2005) The Influence of Intellectual Capital on the types of innovative capabilities, Academy of Management Journal, 48(3), 450-463.

Sundac, D., Krmpotic, F. (2009). Measurement and management of intellectual. Tourism and Hospitality Management, 15(2), 279-290.

Sveiby, K.E. (1997). The New Organizational Wealth: Managing and Measuring Knowledge-Based Assets, New York: Berrett-Koehler.

Tejedo-Romero, F. and Tejada-Ponce, A. (2013). "Incidence of corporate characteristics in the policy of voluntary disclosure of intellectual capital information: evidence in Spanish companies". Tourism \& Management Studies, 9(2) 78-85.

Youndt, M. A., Subramaniam, M., and Snell, S. A. (2004).Intellectual capital profiles: An examination of investments and returns. Journal of Management Studies, 41(2), 335-362.

Wall, A. (2005). The measurement and management of Intellectual Capital in the public Sector: taking the lead or waiting for direction? Public Management Review, 7(2), 289-303.

Wright, P. M., Coff, R., Moliterno, T. P. (2014). Strategic Human Capital Crossing the Great Divide. Journal of Management, 40(2), 353-370.

Wyatt, A. (2008). What financial and non-financial information on intangibles is value-relevant? A review of the evidence. Accounting and Business Research, 38(3), 217-256.

Submetido: 30.06.2014

Aceite: 27.11.2014 